\title{
Utilizing resonant magnetic perturbations to enhance neoclassical tearing mode stabilization by rf current
}

Xiaoguang $\mathrm{Wang}^{1}$,Xiaodong Zhang ${ }^{1}$, Qingquan $\mathrm{Yu}^{2}$, Bin $\mathrm{Wu}^{* 1}$, Sizheng Zhu $^{1}$,Jinfang Wang ${ }^{1}$, Yang Zhang ${ }^{1}$, Xiaojing Wang ${ }^{3}$

${ }^{1}$ Institute of Plasma Physics, Chinese Academy of Sciences, Hefei, China

${ }^{2}$ Max-Planck-Institut für Plasmaphysik, D-85748, Garching, Germany

${ }^{3}$ University of Science and Technology of China, Hefei, China

*Corresponding author email: wubin@ipp.ac.cn

\begin{abstract}
A new method to stabilize the rotating neoclassical tearing mode (NTM) by using both the rf current drive and the static resonant magnetic perturbation (RMP) is investigated. When a non-uniform mode rotation is induced by the RMP, the stabilization of NTM by the rf current is found to be enhanced if the RMP phase has a half period difference from that of the rf wave deposition along the helical angle. The required rf current for mode stabilization is reduced by about one third if an appropriate RMP amplitude is applied.
\end{abstract}

Keywords: NTMs, RMP, rf current, MHD instability

\section{Introduction}

Neoclassical tearing modes (NTMs) can limit the plasma pressure ${ }^{1-6}$, slow down the plasma rotation or even lead to major disruptions of tokamak plasmas ${ }^{7-12}$. These modes therefore have to be stabilized for a fusion reactor. Localized non-inductive rf current drive, such as the electron cyclotron current drive (ECCD), at relevant rational surfaces is an effective method for stabilizing NTMs ${ }^{13-18}$. Electron cyclotron wave (ECW) was already applied to control NTMs successfully in tokamak experiments ${ }^{19-22}$, and it is also planned to be utilized in ITER for this purpose ${ }^{23,24}$.

It is well known that the non-inductive current drive at the o-point (x-point) of magnetic islands along the plasma current direction has a stabilizing (destabilizing)

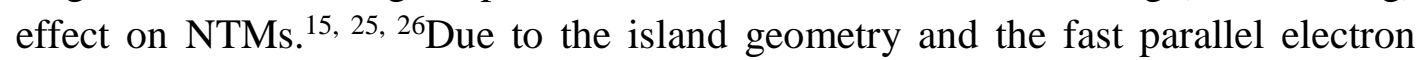
transport, when the radial deposition width of ECW is small, the continuous ECCD has about the same stabilizing effect as the modulated ECCD, which drives the current only around the island's o-point region. ${ }^{15,16}$ If the radial deposition width of ECW is sufficiently large, the modulated ECCD is more effective in stabilizing NTMs than the continuous one. ${ }^{16,27}$ However, the modulated ECCD are carried out only with $50 \%$ on-duty ratio, i.e., the rf current is driven only for half the time when the island's o-point passes through the ECW deposition region. ${ }^{21,27}$

If the island rotation is modulated to slow down (speed up) the rotation when the island's o-point (x-point) passes through the ECW deposition region, the 
stabilizing effect of ECCD on the island will be increased due to a longer time period of the rf current drive around the island's o-point. The resonant magnetic perturbation (RMP) is known to be able to affect the island rotation ${ }^{8,9}, 12,28$, ${ }^{29}$,especially for a fusion reactor plasma ${ }^{28}$. A moderate amplitude of RMP can lead to a non-uniform island rotation without causing mode locking, speeding up the island rotation in half period but slowing down the rotation in another half one.

In this paper the effect of applied static RMPs on the NTM stabilization by ECCD is investigated numerically. It is found that if the applied RMP has half a period phase difference with respect to the ECW deposition region along the helical angle, the island rotates more slowly (fast) when the island's o-point (x-point) passes through the ECW deposition region, leading to an enhanced stabilizing effect on rotating NTMs by ECCD. On the contrary, the stabilizing effect is degraded when the RMP has the same phase as the ECW deposition. The degree of non-uniform island rotation is affected by the RMP amplitude. A too large RMP amplitude will result in mode locking. It will be shown that, if an appropriated RMP amplitude and phase is applied, the required driven current for NTM stabilization is reduced by about one third. For a fusion reactor a few tens of MWs of ECW power is expected to be required to stabilize a single NTM, depending on the radial ECW deposition width and the value of the local bootstrap current density at the resonant surface. Utilizing RMPs could significantly reduce the ECW power requirement for the mode stabilization.

The theoretical mode for our numerical calculations is introduced in the second section. And numerical results on the stabilization of NTM by the combination ECCD and RMPs are reported in Sec. 3. The discussion and summary are given in Sec. 4.

\section{Theoretical model}

The basic equations utilized for our numerical calculations are Ohm's law, the equation of motion and the energy conservation equation. The large aspect-ratio tokamak approximation is made. The magnetic field is defined as $\boldsymbol{B}=B_{t} \boldsymbol{e}_{t}$ $(k r / m) B_{t} \boldsymbol{e}_{\theta}+\nabla \psi \times \boldsymbol{e}_{t}$, where $\psi$ is the helical flux function, $m / r$ and $k=n / R$ are the wave vectors in the $\boldsymbol{e}_{\theta}$ (poloidal) and $\boldsymbol{e}_{t}$ (toroidal) directions, $\mathrm{r}$ and $R$ are the minor and the major radius, and $m$ and $n$ are the poloidal and toroidal mode numbers. Normalizing the length to the minor $a$, the time to the resistive time $\tau_{R}=a^{2} \mu_{0} / \eta$, the helical flux $\psi$ to $a B_{t}$, the plasma velocity $v$ to $a / \tau_{R}$, and the electron temperature $T_{e}$ to its value at the magnetic axis, these equations become

$$
\begin{gathered}
\frac{d \psi}{d t}=E-\eta\left(j-j_{b}-j_{d}\right) \\
\frac{d U}{d t}=S^{2} \nabla_{\|} j+\mu_{\perp} \nabla^{2} U+S_{\mathrm{m}} \\
n_{e} \frac{3 d T_{e}}{2 d t}=\nabla \cdot\left(\chi_{\|} \nabla_{\|} n_{e} T_{e}\right)+\nabla \cdot\left(\chi_{\perp} \nabla_{\perp} n_{e} T_{e}\right)+S_{p}
\end{gathered}
$$


where $d / d t=\partial / \partial t+\boldsymbol{v}_{\perp} \cdot \nabla, \boldsymbol{v}_{\perp}=\nabla \Phi \times \boldsymbol{e}_{\boldsymbol{t}}, j=\nabla^{2} \psi-2 n B_{0 t} /(m R)$ being the plasma current density, $j_{b}=-c_{b}\left(\varepsilon^{1 / 2} / B_{p}\right) n_{e} \frac{d T_{e}}{d r}$ the bootstrap current, $j_{d}$ is the non-inductive current density driven by ECW in $\boldsymbol{e}_{\boldsymbol{t}}$ direction, and $c_{b}$ in the expression of $j_{b}$ is a constant of the order of unity. $\varepsilon=r / R$ is the inverse aspect ratio, $B_{p}$ the poloidal magnetic field, $\mathrm{n}_{\mathrm{e}}$ the electron density, $\eta$ the normalized plasma resistivity, and $\mathrm{E}$ the equilibrium electric field. The Lundquist number $\mathrm{S}=$ $\tau_{\mathrm{R}} / \tau_{\mathrm{A}}$, where $\tau_{A}=a / V_{A}$ is the toroidal Alfvén time, $\tau_{R}=a^{2} \mu_{0} / \eta$ is the resistive time, and $a$ is the minor radius. $U=-\nabla_{\perp}^{2} \phi$ is the plasma vorticity, $\mu$ the plasma viscosity, and $\chi_{\|}$and $\chi_{\perp}$ the parallel and perpendicular heat transport coefficients. $S_{p}$ is the heating power, and $S_{m}$ in equation (2) is the poloidal momentum source which leads to an equilibrium plasma rotation. The plasma density and ion temperature are assumed to be constant here.

The driven current density $j_{d}$ is calculated from the following equation ${ }^{28,30}$,

$$
\frac{\partial j_{d}}{\partial t}=\nabla \cdot\left(\chi_{\| f} \nabla_{\|} j_{d}\right)+\nabla \cdot\left(\chi_{\perp f} \nabla_{\perp} j_{d}\right)+v_{f}\left(j_{e c c d}-j_{d}\right)(4)
$$

where $\chi_{\| f}$ and $\chi_{\perp f}$ are the parallel and perpendicular fast electron transport coefficients, $v_{f}$ the collisional frequency of fast electron, and $j_{e c c d}$ is the source current density driven by ECW,

$$
j_{e c c d}=j_{e c 0} \exp \left(-4\left(\left(r-r_{d s}\right) / w_{d e p}\right)^{2}\right) \Pi\left(\xi_{0}, \Delta \xi_{e c}\right)
$$

In Eq. (5) $j_{e c 0}, w_{d e p}$ and $r_{d s}$ specify the magnitude, the radial full $1 / \mathrm{e}$ width and the radial deposition location of the source. $\Pi\left(\xi_{0}, \Delta \xi_{e c}\right)$ is a square box function for taking into account the wave deposition profile along the helical angle $\xi=m \theta+$ $n \phi$ and the island rotation, which is defined as $\Pi\left(\xi_{0}, \Delta \xi_{\text {ec }}\right)=1$ for $\xi_{\text {on }}<\xi_{0}<\xi_{\text {off }}$, and $\Pi\left(\xi_{0}, \Delta \xi_{E C}\right)=0$ elsewhere, where $\xi_{0}$ is the instantaneous helical angle of the island's o-point, and $\xi_{\text {on }}\left(\xi_{o f f}\right)$ is the helical angle at which the ECW is turned on (off).The ECW deposition region is assumed to be centered at $\xi=0$, with an instantaneous deposition width $\Delta \xi_{e c}=m \Delta \theta+n \Delta \phi$ along the helical angle. The total driven current $I_{c d}$ is obtained by integrating $\mathrm{j}_{\mathrm{d}}$ over the plasma cross section.

The effect of the applied static RMP is taken into account by the boundary condition

$$
\psi_{m / n}(r=a)=\psi_{a} a B_{t} \cos (m \theta+n \phi),
$$

Where $\psi_{a}$ is the normalized amplitude of the $m / n$ component of $\psi$ at $r=a$. The corresponding radial magnetic field at $r=a$ is given by $b_{r a}=m \psi_{a} B_{t}$.

\section{Numerical Results}

We only consider the $m / n=3 / 2$ mode in our calculations. A monotonic profile for the safety factor $\mathrm{q}$ is assumed with the $\mathrm{q}=3 / 2$ surface located at $r_{s}=0.575 \mathrm{a}$, and the local magnetic shear length $L_{q}=q /\left(a q^{\prime}\right)=0.57$. The stability index of $3 / 2$ mode is $\Delta^{\prime} r_{s} \cong-1.16$ at w=0.009a. The Lundquist number is $S=1.0 \times 10^{7}$ at 
$q=1.5$ rational surface. The perpendicular and the parallel transport coefficients of thermal electrons are taken to be $\chi_{\perp} /\left(\tau_{R} / a\right)=30$ and $\chi_{\|} /\left(\tau_{R} / a\right)=3 \times 10^{9}$ at $3 / 2$ rational surface respectively, leading to $w_{d}$ to be about $0.05 a$, where $w_{d}$ is the critical island width above which the electron temperature profile flattens inside the magnetic island. ${ }^{18,31,32}$ The viscous time $\tau_{\mu}=a^{2} / \mu$ has been assumed to be ten times smaller than the resistive time. The equilibrium stream function profile is given by $\phi \sim\left[1-\left(\frac{\mathrm{r}}{\mathrm{a}}\right)^{2}\right]^{3}$. The momentum source $\mathrm{S}_{\mathrm{m}}=-\mu_{\perp} \nabla^{2} U$ is calculated from the equilibrium stream function, to sustain an equilibrium plasma rotation. The local poloidal equilibrium plasma rotation velocity profile can be found in Fig. 10. The normalized equilibrium electron temperature profile is given by $T_{e}=0.95[1-$ $\left.\left(\frac{r}{a}\right)^{2}\right]^{4}+0.05$. The power source $\mathrm{S}_{\mathrm{p}}=-\nabla \cdot\left(\chi_{\perp} \nabla_{\perp} n_{e} T_{e}\right)$ is calculated from the equilibrium electron temperature. The initial mode frequency is $\omega \tau_{R}=3.6 \times 10^{4}$. The effect of the combined ECCD and RMP on the mode amplitude is presented in figure 1 , in which the normalized island width $w / a$ is shown as a function of the normalized time $t / \tau_{R}$ for the local bootstrap current density fraction 0.19 at the rational surface. The initial island width is taken to be about $0.1 a$. Without ECCD, the island width saturates at $w=0.13 a$. After a continuous ECCD is applied at $\xi=0$ and $\mathrm{r}=\mathrm{r}_{\mathrm{s}}$ with $I_{c d}=0.02$ and $w_{d e p}=0.08$, the island width saturates at $0.09 \mathrm{a}$. The following input parameters are taken: the collision frequency of fast electrons $v_{\mathrm{f}} \tau_{\mathrm{R}}=3 \times 10^{3}, \chi_{\| \mathrm{f}}=10 \chi_{\|}, \chi_{\perp \mathrm{f}}=\chi_{\perp}$, and the instantaneous wave deposition width $\Delta \xi_{e c}=0.23 \pi$. After the island width reaches a steady value, RMPs with two different helical phases, $\psi_{\mathrm{a}}=1 \times 10^{-6}$ and $\psi_{\mathrm{a}}=-1 \times 10^{-6}$, are further applied. The RMP with a positive (negative) amplitude, $\psi_{\mathrm{a}}=1 \times 10^{-6} \quad\left(\psi_{\mathrm{a}}=-1 \times\right.$ $10^{-6}$ ), will generate a magnetic island with its o-point's at a helical angle $\xi_{\mathrm{RMP}}=$ $0\left(\xi_{\mathrm{RMP}}=\pi\right)$ under the vacuum assumption or tend to lock an existing island's opoint at a helical angle $\xi_{\mathrm{RMP}}=0\left(\xi_{\mathrm{RMP}}=\pi\right)$. Hereafter the parameter $\xi_{\mathrm{RMP}}$ is utilized to describe the phase of the applied RMP along the helical angle. It is seen from figure 1 that the island width increases for $\xi_{\mathrm{RMP}}=0$ compared to that without RMP but decreases for $\xi_{\text {RMP }}=\pi$. This is due to the non-uniform island rotation caused by the RMP as shown below. The applied RMP amplitude is quite small, so that it has little effect on the island width without ECCD, and the island width is determined by the perturbed bootstrap current, the rf current and the equilibrium plasma current density profile. 


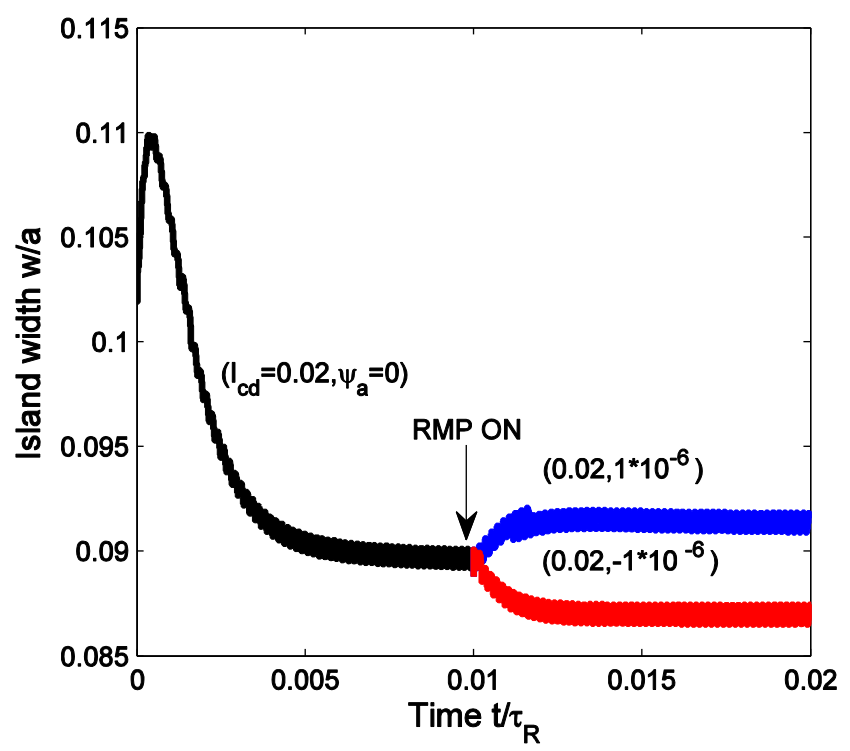

Figure 1. The normalized island widths w/a are demonstrated as a function of the normalized time $\mathrm{t} / \tau_{\mathrm{R}}$, by applying ECCD at $\mathrm{t}=0$ with $\mathrm{I}_{\mathrm{cd}}=0.02$ and $w_{\text {dep }}=$ 0.08 . After the steady state is reached, RMPs with $\psi_{\mathrm{a}}=1 \times 10^{-6}$ and $\psi_{\mathrm{a}}=$ $-1 \times 10^{-6}$ are further applied. The island width increases for $\psi_{\mathrm{a}}=1 \times 10^{-6}$ but decreases for $\psi_{\mathrm{a}}=-1 \times 10^{-6}$.

Corresponding to figure 1, Fig.2presents the time evolution of the mode frequency and the phase of the island's o-point in the time period between $\mathrm{t}=$ $0.0119 \tau_{\mathrm{R}}$ andt $=0.0122 \tau_{\mathrm{R}}$ for $\psi_{a}=1 \times 10^{-6}$ (a) and $\psi_{a}=-1 \times 10^{-0}$ (b), i.e., $\xi_{R M P}=0$ (a) and $\xi_{R M P}=\pi(\mathrm{b})$. The non-uniform island rotation can be described by the parameter

$$
\mathrm{u}_{\mathrm{f}}=\frac{\left(\omega_{\max }-\omega_{\min }\right)}{\left(\omega_{\max }+\omega_{\min }\right) / 2}
$$

where $\omega_{\max }$ and $\omega_{\min }$ are the maximum and the minimum mode rotation frequency in one rotation period. It is seen from Fig. 2 that $\mathrm{u}_{\mathrm{f}} \approx 0.13$ for both $\psi_{\mathrm{a}}=1 \times 10^{-6}$ and $\psi_{\mathrm{a}}=-1 \times 10^{-6}$. For $\xi_{\mathrm{RMP}}=0(\pi)$, the island's o-point rotates faster (more slowly) at $\xi=0$ where the ECW is deposited. This can also be clearly seen from the time intervals $\Delta t_{1}$ and $\Delta t_{2}$ marked in figure 2, where $\Delta t_{2}$ is the time interval for the island's o-point to rotate from $-\pi / 2$ to $\pi / 2$, while $\Delta \mathrm{t}_{1}$ is for the island's o-point to rotate from $\pi / 2$ to $-\pi / 2 . \Delta t_{2}$ is smaller (larger) than $\Delta t_{1}$ for $\xi_{\text {RMP }}=0(\pi)$. As a result, the o-point (x-point) stays for a longer period of time in the ECW deposition region for $\xi_{\mathrm{RMP}}=\pi(0)$, leading to enhanced (degraded) mode stabilization shown in figure 1.

From Fig. $2 b$ it is seen that $\xi_{s}$, the helical angle of island's o-point at which the island rotation is the slowest, is $-0.10 \pi$ rather than zero for $\xi_{\mathrm{RMP}}=\pi$. When the equilibrium plasma rotation direction is reversed in our calculation, it is found that $\xi_{\mathrm{s}}=0.10 \pi$ as expected, since the phase lag is due to plasma viscous effect, i.e., the second term on the right hand side of Eq. 2. 

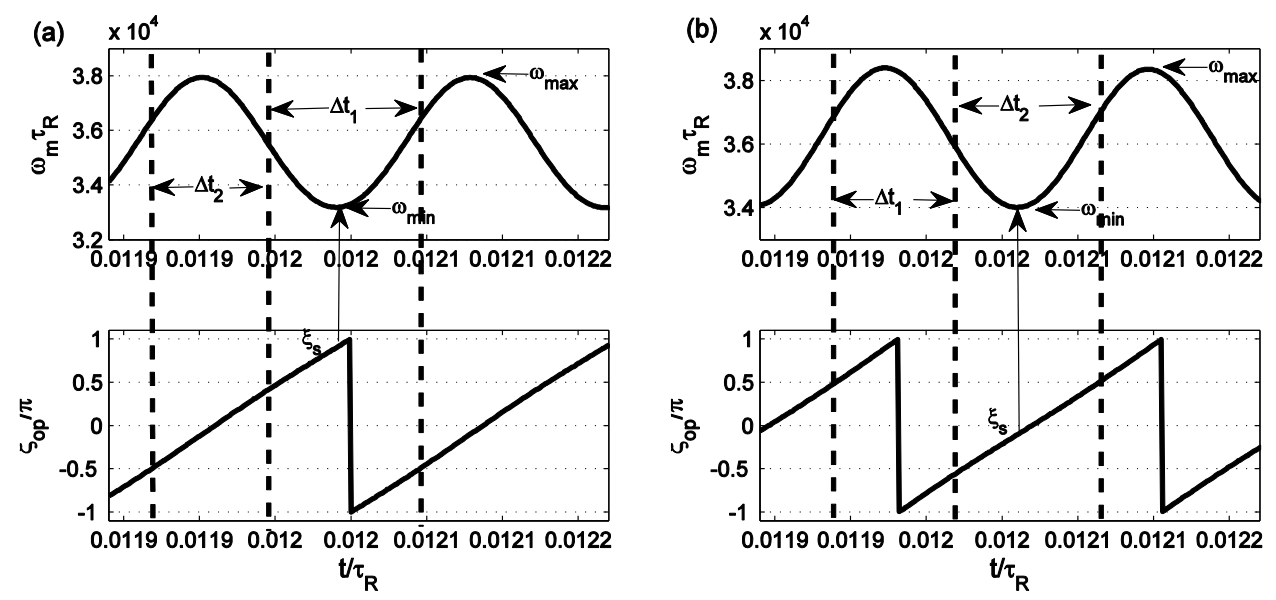

Figure 2 Time evolution of the mode frequency and the phase of the island's opoint are shown for $\psi_{\mathrm{a}}=1 \times 10^{-6}$ (a) and $\psi_{\mathrm{a}}=-1 \times 10^{-6}$ (b). The time interval $\Delta t_{2}$ is for the island's o-point to rotate from $-\pi / 2$ to $\pi / 2$, while $\Delta t_{1}$ is for the island's o-point to rotate from $\pi / 2$ to $-\pi / 2 . \Delta \mathrm{t}_{2}$ is smaller (larger) than $\Delta \mathrm{t}_{1}$ for $\psi_{\mathrm{a}}=1 \times 10^{-6}\left(\psi_{\mathrm{a}}=-1 \times 10^{-6}\right)$, i.e., $\xi_{\mathrm{RMP}}=0(\pi) . \omega_{\max }$ and $\omega_{\min }$ are the maximum and the minimum mode rotation frequency in one period. $\xi_{\mathrm{s}}$ is the phase of island's o-point when the island rotation is the slowest.

Corresponding to Fig.1, Fig. 3 shows the saturated island width, $w_{\text {sat }}$, as function of the RMP phase. Without RMP $w_{\text {sat }}=0.0892 a$ as shown by the horizontal dotdashed line. The RMP improves the NTM stabilization by ECCD for $0.6 \pi<$ $\xi_{\mathrm{RMP}}<1.6 \pi$. The saturated width is smallest for $\xi_{\mathrm{RMP}}=1.1 \pi$. As the difference in the island width between the two cases, $\xi_{R M P}=\pi$ and $\xi_{R M P}=1.1 \pi$, is small, only $\xi_{\mathrm{RMP}}=\pi$ will be considered in the following results to further look into the enhanced stabilizing effect caused by the RMP.

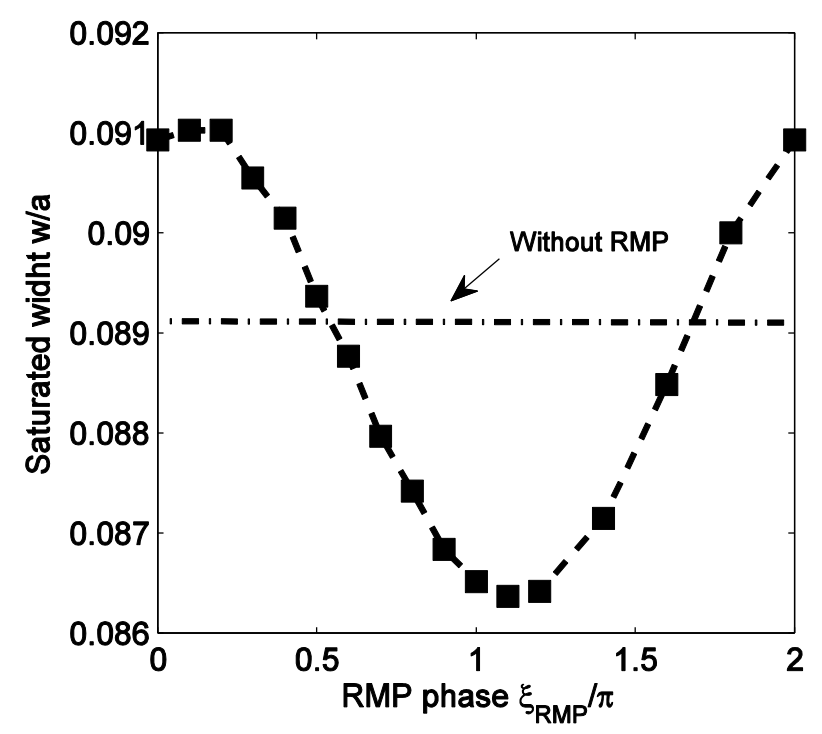

Figure 3. Saturated island widths as a function of RMP phase $\xi_{\mathrm{RMP}}$ are presented for $\mathrm{I}_{\mathrm{cd}}=0.02, w_{\text {dep }}=0.08$ and $\left|\psi_{\mathrm{a}}\right|=1 \times 10^{-6}$. The horizontal dot-dashed line shows the saturated width without RMP. 
Obviously, the degree of non-uniform island rotation is affected by the RMP amplitude. In Fig.4 the island widths are shown as a function of time for different RMP amplitude. Other input parameters are the same as those for Fig.1. It is seen that the saturated island width first decreases with increasing the RMP amplitude due to a more non-uniform island rotation caused by a stronger RMP. For a sufficiently large RMP amplitude, $\psi_{a}=-4 \times 10^{-6}$, the island width first decreases and then increases up to $0.16 a$, since the island's $\mathrm{x}$-point is locked by the RMP at $\xi=0$ where the ECW is deposited. It is known that applied ECCD at island's x-point is destabilizing.

Corresponding to Fig. 4, Fig. 5 shows the time evolution of the mode frequency and the phase of island's O-point in the time interval from $0.011 \tau_{R}$ to $0.0113 \tau_{R}$. The island rotation becomes more non-uniform for a larger RMP amplitude, and $\mathrm{u}_{\mathrm{f}}$ increase from 0.12 to 0.58 . Fig. 6 shows the mode frequency and the o-point phase in a later time interval for $\psi_{\mathrm{a}}=-4 \times 10^{-6}$. The mode frequency eventually decreases to zero due to mode locking. The island's o-point is locked at $\xi=\pi$ (the $\mathrm{x}$-point is locked at $\xi=0)$. It is therefore clear that only the RMP with an appropriate amplitude can enhance the stabilization of rotating NTMs by ECCD for $\xi_{\mathrm{RMP}}=\pi$.

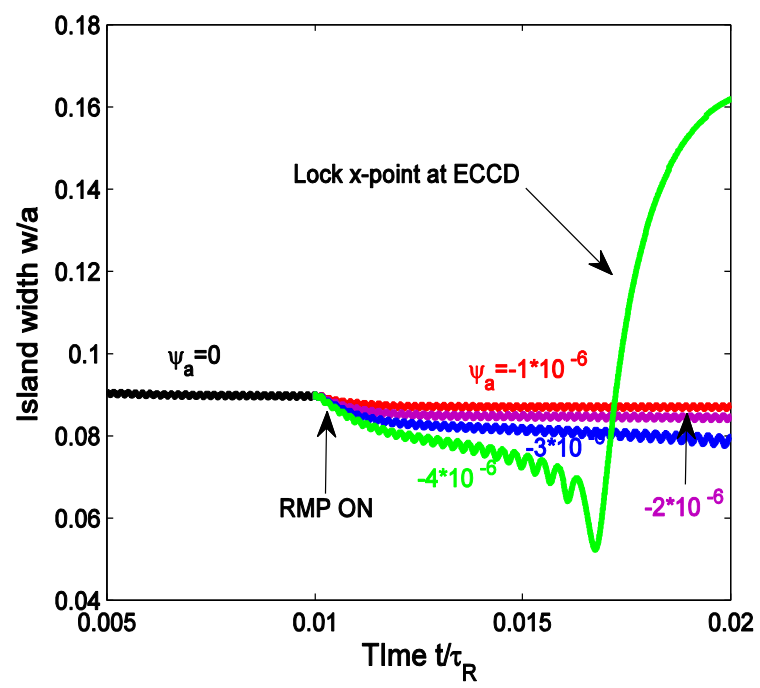

Figure 4. Time evolution of island widths are shown for $\mathrm{I}_{\mathrm{cd}}=0.02, w_{\text {dep }}=$ 0.08 with different RMP amplitude and $\xi_{\text {RMP }}=\pi$. The saturated island width decreases with increasing RMP amplitude if the amplitude is not too large. For $\Psi_{\mathrm{a}}=-4 \times 10^{-6}$ the island's $\mathrm{x}$-point is locked by the RMP at $\xi=0$ where the ECW is deposited. 


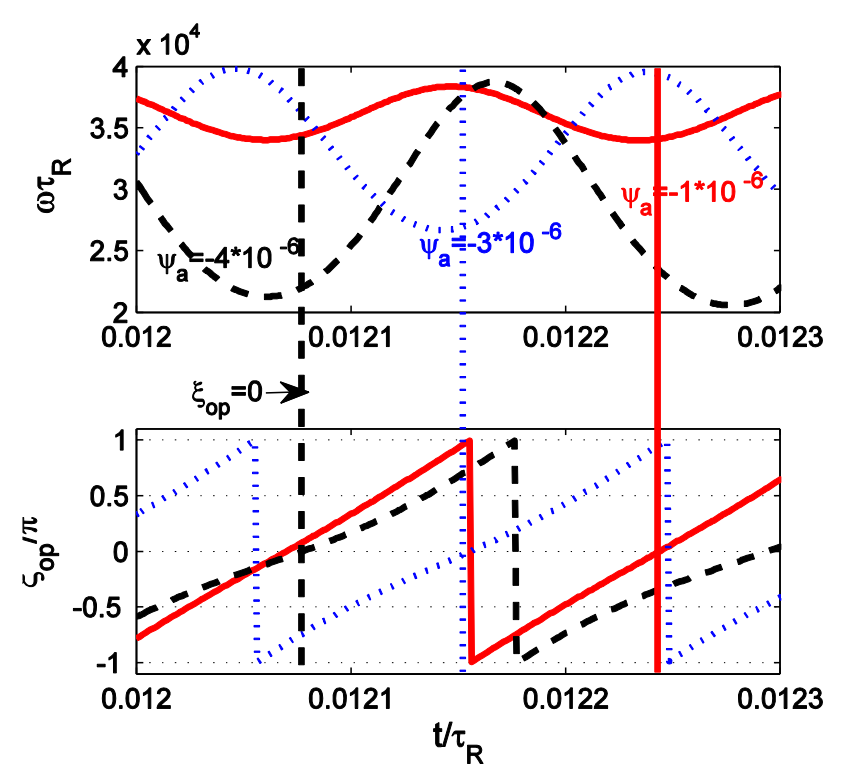

Figure 5. Corresponding to Fig. 4, the time evolution of the mode frequency and the phase of island's o-point are presented at a time interval before mode locking for different RMP amplitude. The mode rotation becomes more non-uniform with increasing RMP amplitude. The three vertical solid lines indicate the location of island's o-point.
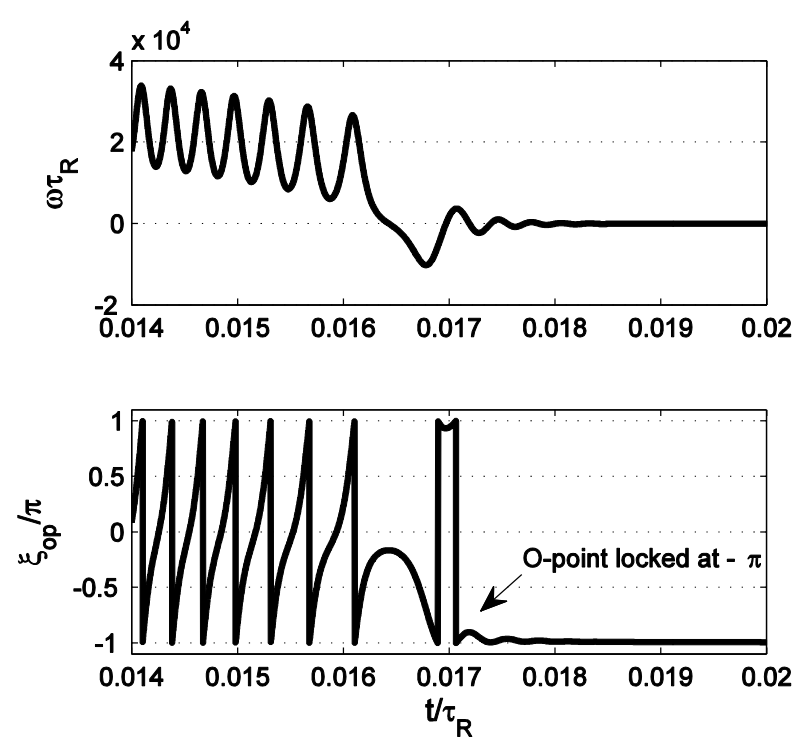

Figure 6. Corresponding to the $\psi_{a}=-4 \times 10^{-6}$ case in Fig. 4 , time evolution of the mode frequency and phase of island's o-point are shown. The mode frequency decreases to zero after mode locking, and the island's o-point is locked at $\xi=\pi$. 

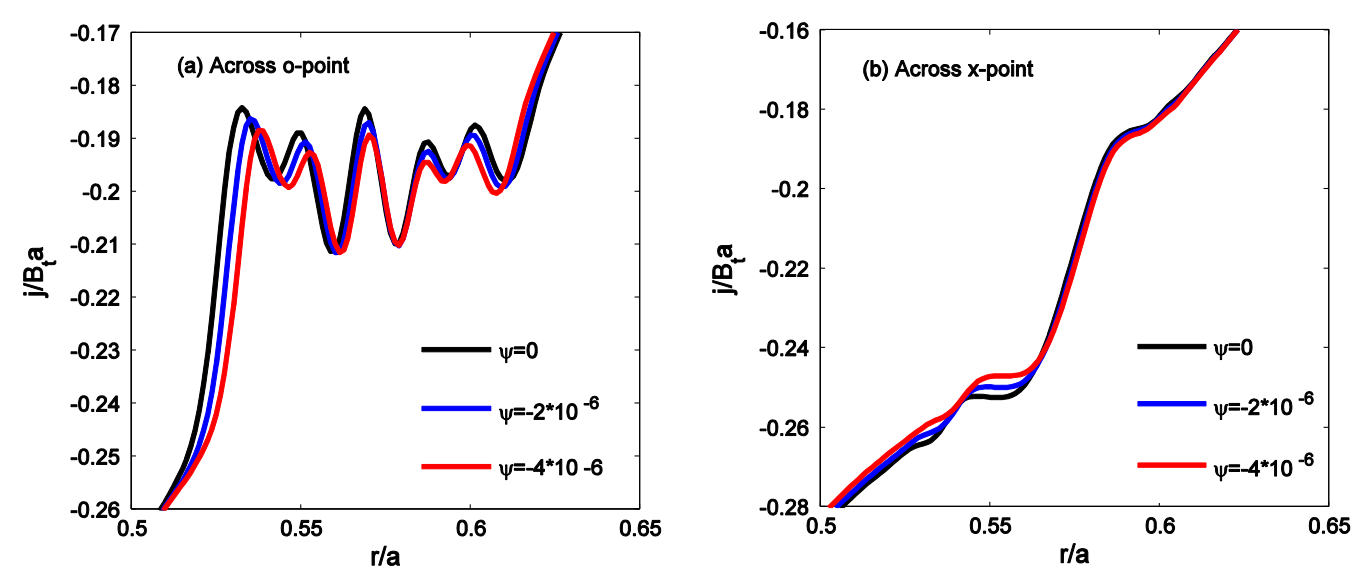

Figure 7. Corresponding to Fig.4, the radial profiles of the normalized plasma current density $j(r) / B_{t} a$ at the helical angle passing through the island's o-point (a) and x-point (b) are shown for different RMP amplitude, when the island o-point is at $\xi=0$. The black line wihtout RMP is chosen at the time $t=0.007 \tau_{R}$. The cases with RMP are at $t=0.012 \tau_{R}$.

Corresponding to Fig. 4, Fig. 7 shows the radial profiles of the normalized plasma current density profile $j(r) / B_{t} a$ at the helical angle passing through the island's o-point (a) and x-point (b) for different RMP amplitude, when the o-point is at $\xi=0$. The black line wihtout RMP is chosen at $t=0.007 \tau_{R}$. The cases with RMP are at $t=0.012 \tau_{R}$. The negative values in the figures represent that the current density is along the negative $\mathbf{e}_{t}$ direction, i.e., the direction for the equilibrium plasma current density utilized for the calculations. It is seen from Fig. 7 that the amplitude of the plasma current density around island's o-point (x-point) increase (decreases) with the RMP amplitude due to the more non-uniform mode rotation caused by stronger RMP. This explains why the island width decreases with increasing the RMP amplitude for $\xi_{\mathrm{RMP}}=\pi$.

With a larger driven current amplitude, $\mathrm{I}_{\mathrm{cd}}=0.025$, while keeping other parameters unchanged, the normalized island widths are demonstrated as a function of the normalized time in figure 8 for different amplitudes of RMP, (a) $\psi_{a}=-1 \times$ $10^{-6}$ to $-5 \times 10^{-6}$ and (b) $-6 \times 10^{-6}$ to $-9 \times 10^{-6}$. It is seen that the island is stabilized to a small island width for $\psi_{\mathrm{a}}=-4 \times 10^{-6}$ to $-8 \times 10^{-6}$. The required time for mode stabilization decreases with increasing RMP amplitude. When the RMP amplitude is too large, $\psi_{\mathrm{a}}=-9 \times 10^{-6}$ as shown in Fig.8b, the island's $\mathrm{x}$ point is locked at the ECW deposition region at $\xi=0$, and the island width significantly increases similar to that shown before. A parameter window of the RMP amplitude, from $\psi_{\mathrm{a}}=-4 \times 10^{-6}$ to $-8 \times 10^{-6}$, exists for the mode stabilization by the RMP without causing mode locking. For $\psi_{\mathrm{a}}=-9 \times 10^{-6}$, the mode can also be stabilized if the RMP is turned off before mode locking when the island width is decreased to $\mathrm{w}=0.05 \mathrm{a}$, as also shown in Fig. $8 \mathrm{~b}$, since the destabilizing effect of the perturbed bootstrap current becomes weaker for a smaller island width due to the finite ratio between the parallel and perpendicular transport coefficients.

Corresponding to Fig. 8, Fig.9 shows the mode frequency and phase of the 
island's o-point for $\psi_{\mathrm{a}}=-5 \times 10^{-6}$. The o-point is finally locked at $\xi=-0.54 \pi$. This is usually the case for a small locked island, being different from that of a large locked island. ${ }^{29}$
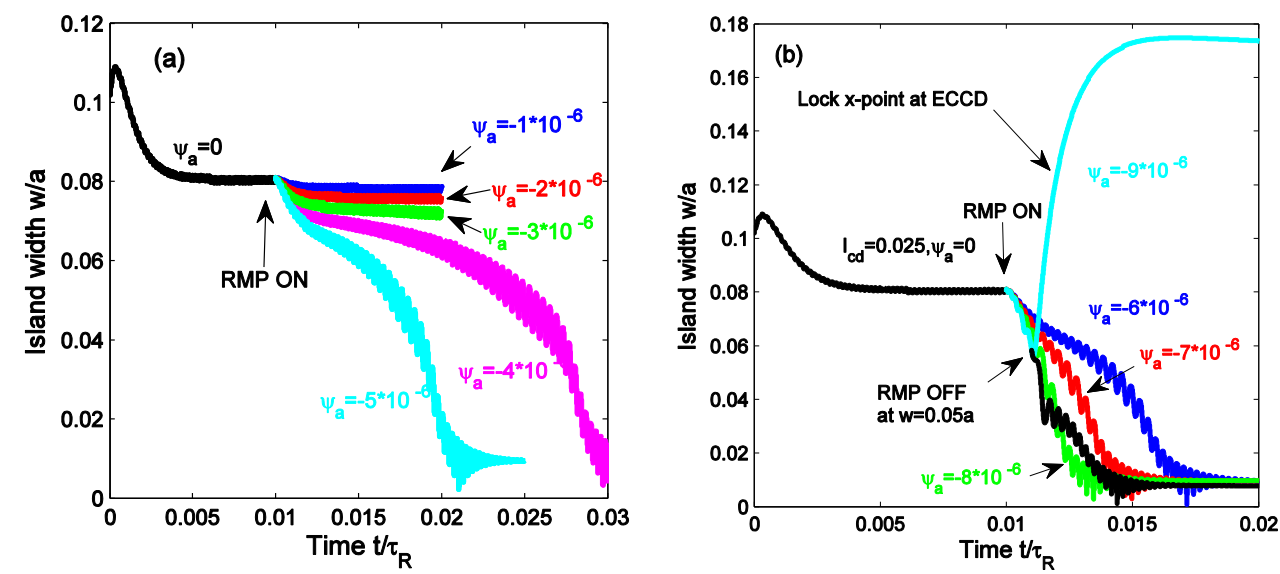

Figure 8. Similar to Fig. 4 but for $\mathrm{I}_{\mathrm{cd}}=0.025$, the time evolution of the island width is shown for different RMP amplitude, (a) from $\psi_{\mathrm{a}}=-1 \times 10^{-6}$ to $-5 \times$ $10^{-6}$ and (b) from $\psi_{\mathrm{a}}=-7 \times 10^{-6}$ to $-9 \times 10^{-6}$. For $\psi_{\mathrm{a}}=-4 \times 10^{-6}$ to $-8 \times 10^{-6}$, the NTM is stabilized. For $\psi_{\mathrm{a}}=-9 \times 10^{-6}$ mode is locked to the RMP. However, in this case the mode can also be stabilized if the RMP is turned off when the island width is decreased to $\mathrm{w}=0.05 a$.
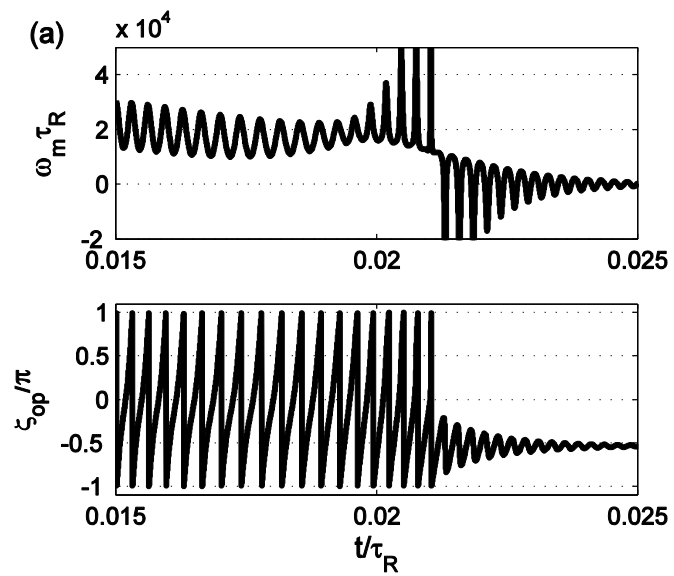

Figure9. Corresponding to the $\psi_{\mathrm{a}}=-5 \times 10^{-6}$ cases in Fig. 8, the time evolution of the mode frequency and phase of island's o-point are shown.

Fig.10 presents the radial profiles of the $m / n=0 / 0$ component poloidal plasma velocity at different time for $\psi_{\mathrm{a}}=-5 \times 10^{-6}$ (a) and $\psi_{\mathrm{a}}=-9 \times 10^{-6}(\mathrm{~b})$. The dashed lines show the equilibrium velocity profile. In Fig. 10a the dot-dashed curve at $\mathrm{t}=0.025 \tau_{\mathrm{R}}$ is the velocity profile after mode stabilization. The plasma rotation velocity is somewhat slowed down by the static RMP due to the small locked island. For the large locked island case as shown by the dot-dashed curve in Fig. 10b, the plasma rotation velocity around rational surface is slowed down to zero after mode locking, as expected. 

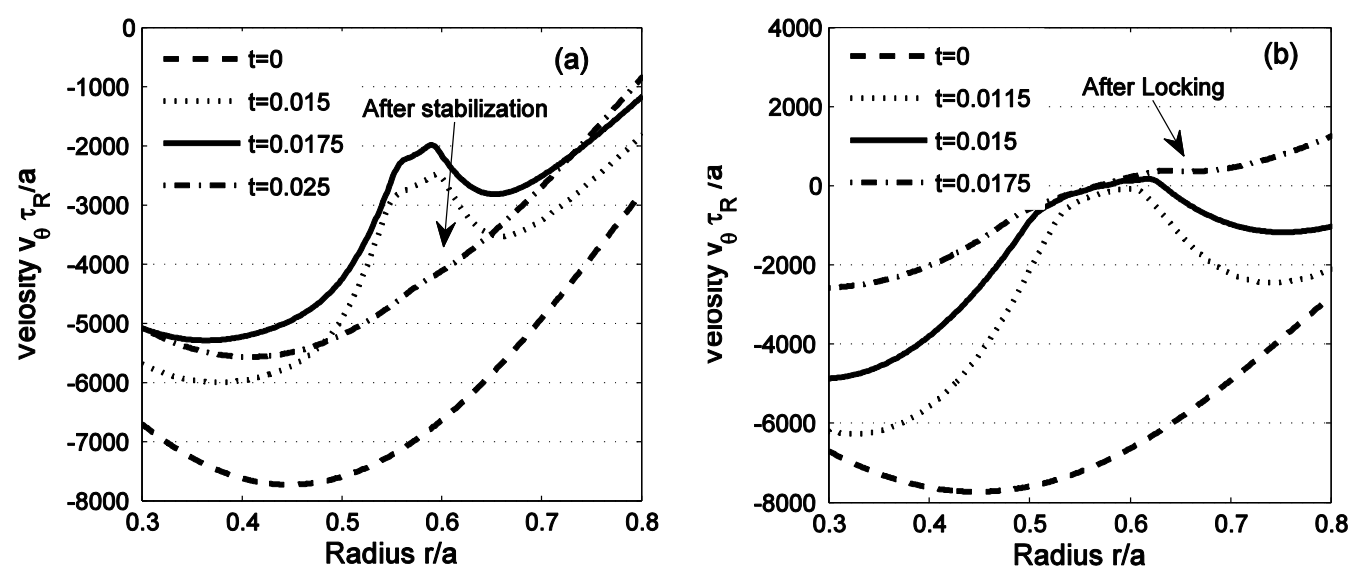

Figure 10. Corresponding to Fig. 8 , radial profiles of the $\mathrm{m} / \mathrm{n}=0 / 0$ component poloidal plasma velocity at different time for $\psi_{\mathrm{a}}=-5 \times 10^{-6}$ (a) and $\psi_{\mathrm{a}}=-9 \times$ $10^{-6}$ (b). The dashed curves show the equilibrium velocity profile. The dot-dashed lines are after mode stabilization (a) and mode locking (b).

Fig.11 shows the island width as a function of time for different amplitude of the driven current with and without applying RMP. The RMP amplitude is chosen to be slightly below the mode locking threshold. The island is stabilized to a small width by continuous current drive for $\mathrm{I}_{\mathrm{cd}}=0.034$ without applying RMP. This value is reduced to $\mathrm{I}_{\mathrm{cd}}=0.022$ if a $\mathrm{RMP}$ with $\psi_{\mathrm{a}}=-4 \times 10^{-6}$ is applied. For this case there is a fast decrease in island width around $t=0.02$, since at that time the island rotation velocity is reduced to $10 \%$ of its initial value when the island's o-point passes through the ECW deposition phase. The required driven current is reduced by about $35 \%$ for mode stabilization when an appropriate RMP amplitude is applied.

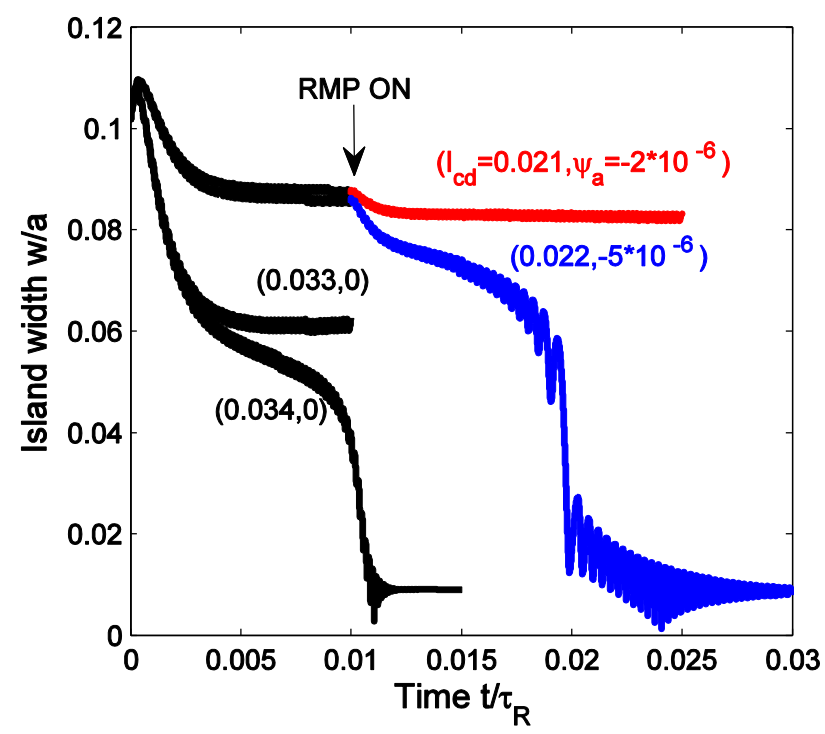

Figure 11. Island width as a function of normalized time is given for different amplitude of the driven current and RMPs. Without applying RMP, $\mathrm{I}_{\mathrm{cd}}=0.034$ is required to stabilize the mode. When an appropriate RMP amplitude is applied, only $\mathrm{I}_{\mathrm{cd}}=0.022$ is required, reducing by about $35 \%$. 
Similar to Fig. 11, calculations with 50\%-modulated ECCD have also been carried out, and essentially the same results as those shown in figure 11 are obtained due to the small ECW deposition width. The required driven current for NTM stabilization is also found to be reduced by about 35\% when an appropriate RMP is applied.

With a larger radial ECW deposition width, $w_{\text {dep }}=0.12$, the time evolution of the island width are shown for continuous (a) and 50\% modulated (b) ECCD respectively in figure 12. Other parameters are kept unchanged. For continuous ECCD, $I_{c d}=0.065$ is required to stabilize the $3 / 2$ NTM without applying RMP. For $\mathrm{I}_{\mathrm{cd}}=0.042$ the saturated island width is $w=0.085 a$ without RMP but is stabilized when $\psi_{\mathrm{a}}=-5 \times 10^{-6}$ is applied. The required driven current for mode stabilization is also reduced by about $35 \%$ with applying RMP. For the case with 50\% modulated ECCD as shown in Fig. $12 \mathrm{~b}, \mathrm{I}_{\mathrm{cd}}=0.056$ is required for mode stabilization without RMP, and this value is reduced by $35 \%$ to $\mathrm{I}_{\mathrm{cd}}=0.038$ with RMP. It is seen that the combined continuous ECCD and RMP is more efficient in stabilizing the NTM than the pure modulated current drive. The combination of modulated ECCD and RMP is the most efficient way for mode stabilization, and the required driven current is about $42 \%$ less than the continuous ECCD alone.
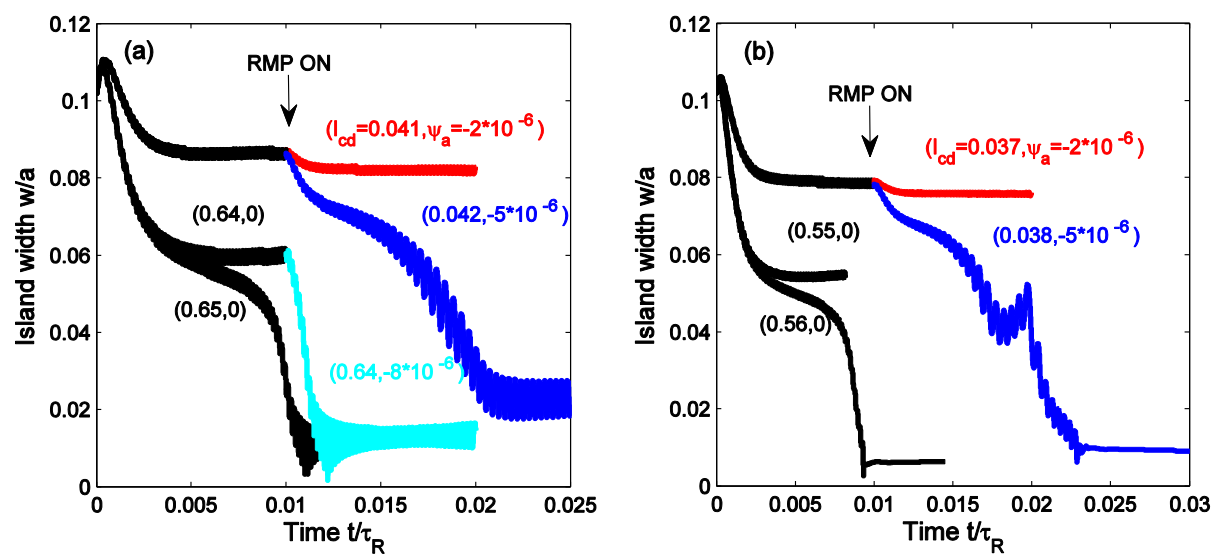

Figure 12. Island widths as a function of normalized time are given for (a) continuous and (b) $50 \%$ modulated ECCD with $w_{\text {dep }}=0.12$. Without applying $R M P, I_{c d}=0.065$ is required to stabilize the NTM for continuous ECCD. When RMPs are applied, $\mathrm{I}_{\mathrm{cd}}=0.042$ is required, reducing by about $35 \%$. For the $50 \%$ modulated ECCD, $\mathrm{I}_{\mathrm{cd}}=0.056$ is required for mode stabilization without RMP.

This value is reduced toI $\mathrm{cd}_{\mathrm{c}}=0.038$ with the RMP.

It is should be noted that for our results the change of the island rotation comes from the change of the poloidal plasma rotation by the poloidal electromagnetic force. In tokamaks the plasma rotation is usually in the toroidal direction in the central region. In this case a much larger RMP amplitude is required to induce a significant non-uniform island rotation, since the electromagnetic force to slow down the island rotation in the toroidal direction is smaller by a factor $(n / m)\left(r_{s} / R\right)$ compared with that in the poloidal direction. In addition, to have the same mode frequency, the 
toroidal rotation speed should be larger by a factor $(\mathrm{m} / \mathrm{n})\left(\mathrm{R} / \mathrm{r}_{\mathrm{s}}\right)$ than the poloidal one. These two effects lead to a larger ratio between the viscous and the electromagnetic force, by a factor $\left[(\mathrm{m} / \mathrm{n})\left(\mathrm{R} / \mathrm{r}_{\mathrm{s}}\right)\right]^{2}$ for the toroidal rotation case. ${ }^{28}$

A smaller Lundquist number $\mathrm{S}$ corresponds to a smaller Alfven velocity in our calculations, resulting in a smaller electromagnetic force. ${ }^{28}$ Fig. 13presents the time evolution of the island width with a 10 times smaller $\mathrm{S}, \mathrm{S}=1 \times 10^{6}$, for $\mathrm{I}_{\mathrm{cd}}=0.03$. In this case a much larger RMP amplitude is required to cause non-uniform island rotation and to improve the stabilization effect, as expected. The enhancement in the mode stabilization by the RMP is also seen. For $\psi_{\mathrm{a}}=-3 \times 10^{-4}$ modelocking occurs during the decrease of the island width, but the NTM is still stabilized if the RMP is turned off at a small island width before mode locking, similar to that shown in figure $8 \mathrm{~b}$. A smaller $\mathrm{S}$ value corresponds to a larger resistive layer width. Once the island width is comparable to or smaller than the resistive layer width, the applied RMP will not drive a regularly non-uniform island rotation as that shown in figure 2, since the island rotation can decouple from the plasma rotation. For high temperature tokamak plasmas, the value of $\mathrm{S}$ is in the order of $10^{7}-10^{8}$ or even larger, and the resistive layer width is smaller than the marginal island width, below which the island is stabilized due to the ion polarization current or the finite ratio between the parallel and the perpendicular transport coefficients. ${ }^{33}$ In our model the ion polarization current associated with the diamagnetic drift has not been included.

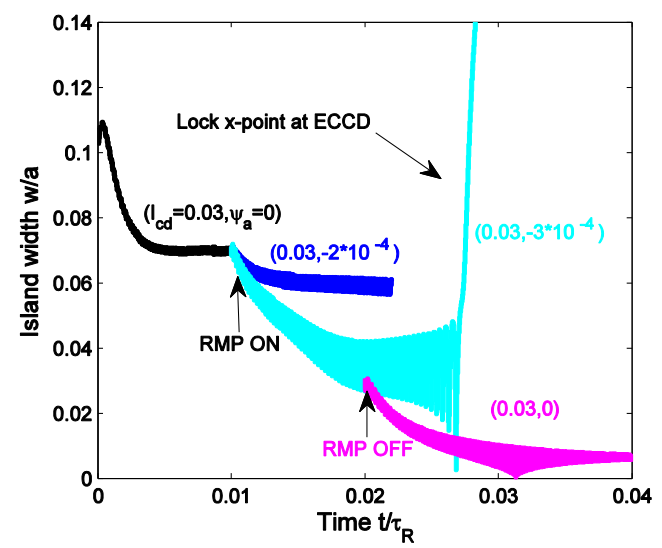

Figure 13.Time evolution of island widths are presented for $\mathrm{I}_{\mathrm{cd}}=0.03, w_{d e p}=$ 0.08 and $S=1 \times 10^{6}$. For $\psi_{\mathrm{a}}=-3 \times 10^{-4}$ the mode is locked to the RMP during the island width decrease if the RMP is always turned on, but it is stabilized if the RMP is turned off at a small island.

Calculations with a larger plasma viscosity have also been carried out. In this case a larger RMP amplitude is required to cause a significant non-uniform island rotation, as expected, and similar improvement in the mode stabilization by the RMP is found.

\section{Discussion and summary}


The stabilization of rotating NTM by the combination of ECCD and RMP is studied in this paper. Due to the non-uniform island rotation caused by the RMP, the island's o-point rotates more slowly when passing through the ECW deposition region along helical angle, if the RMP phase has a half period difference from that of the ECW deposition region. Such a RMP can therefore enhance the mode stabilization by ECCD. The enhancement increases with RMP amplitude if it is below the mode locking threshold. The required driven current for mode stabilization is reduced by about $35 \%$ by using an appropriate RMP amplitude. For a too large RMP amplitude the island's X-point is locked at ECW deposition region, and ECCD is actually destabilizing after mode locking. Feedback control of the RMP amplitude could maximize the enhancement by the applied RMP while avoid mode locking.

As found earlier, the effect of RMP on island rotation depends on the RMP amplitude, the island width, the original island rotation frequency, plasma viscosity, and the Alfven velocity. ${ }^{28}$ Being different from the existing tokamak plasmas, a much smaller error field, about $b_{\mathrm{ra}} / \mathrm{B}_{0 \mathrm{t}}=10^{-5}$ or smaller, is predicted to be able to lock the magnetic island in a fusion reactor. ${ }^{28}$ This suggests that applied RMPs from the error field correction coils are necessary to correct the intrinsic error field, which is usually in the order of $\mathrm{b}_{\mathrm{ra}} / \mathrm{B}_{0 \mathrm{t}}=10^{-4}$, to an acceptable level and to a right phase with respect to the ECW deposition region in order to reduce the required driven current for NTM stabilization. Although our calculations are only for the $\mathrm{m} / \mathrm{n}=3 / 2$ mode, same mechanism exists for the $2 / 1$ or other modes.

In previous numerical simulations ${ }^{28}$ and experiments ${ }^{34}$, studies have been carried out by using the RMP to directly lock the island's o-point at the phase of ECW deposition. The improvement of mode stabilization by ECCD is also observed for a sufficiently high bootstrap current density fraction. However, with this method the local plasma rotation is reduced to zero due to mode locking, which might be unfavorable since the plasma rotation is known to be important in determining the plasma confinement.

The driven current by ECWis given by $\mathrm{I}_{c d}=\gamma_{e c} \mathrm{P}_{\mathrm{ec}} / \mathrm{n}_{\mathrm{e}} \mathrm{R},{ }^{35}$ where $\gamma_{e c}$ is the current drive efficiency, and $\mathrm{P}_{\mathrm{ec}}$ is the ECW power. For a fusion reactor like ITER with $\mathrm{R}=6.2 \mathrm{~m}, \mathrm{n}_{\mathrm{e}}=10^{20} \mathrm{~m}^{-3}$, the plasma current $\mathrm{I}_{\mathrm{p}}=15 \mathrm{MA}$, and $\gamma_{e c}=0.3 \times$ $10^{19} \mathrm{Am}^{-2} \mathrm{~W}^{-1}, 35$ one finds that $\mathrm{P}_{\mathrm{ec}}=30 \mathrm{MWs}$ if only $\mathrm{I}_{\mathrm{cd}} / \mathrm{I}_{\mathrm{p}}=0.01$ is required for the NTM stabilization. If the required rf current is reduced by one third, then 10MWs ECW power is saved. As the island growth time depends on the plasma resistivity and will be a quite long time period (about 100s) in a fusion reactor, ${ }^{3}$ the feedback control of slowly changing RMP amplitude is easy from the technical point of view, and in this case the required power to drive extra current in the saddle coils is estimated to be much less than 10MWs. As mentioned above, the required RMP amplitude to cause a non-uniform NTM rotation is smaller than that required to correct the intrinsic error field in a fusion reactor. ${ }^{28}$ This means that neither extra coils nor lager current in the saddle coils is required. On the contrary, the error field should not be completely corrected such that the remaining parts, having an appropriate spectrum and phase, can sever as the required RMP to enhance the NTM stabilization. The reduction in cost of the ECCD current should be much larger than 
the cost of phasing the saddle coil current in an appropriate way, even if neglecting the additional cost saved for building more ECW systems and the windows to be opened in the vacuum vessel and blanket for more ECW launchers. Obviously, due to the requirement of tritium self-sufficiency for a fusion reactor, the windows in a reactor should be as less as possible.

It is well known that RMPs can also be utilized for ELMs control, ${ }^{36-38}$ which often leads to somewhat confinement degradation (density pump out). ${ }^{39,40}$ Usually only the RMP components with high poloidal numbers generated by the so-called ELM coils are required for ELM control, although the low-m components will also be generated simultaneously. As both the ELMs coils and the error field correction coils will be built in ITER, both coils can be utilized for optimizing the spectrum of the low-m components. Once the NTM is stabilized, reducing the island width to be below the marginal island width, the low-m component of RMPs for enhancing the NTM stabilization can be turned off. Therefore, afterwards it will not degrade confinement but improves it due to NTM stabilization. Our results suggest that it is better to take into account the requirements from both the ELM and NTM control in designing the ELMs coils and the error field correction coils in a fusion reactor, being able to generate appropriate spectrum and phase of RMPs for both purposes.

For the RMP spectrum with a main component, e.g., the $m / n=3 / 2$ component, other components such as $m=2 / 2$ and $4 / 2$ components will also be there. The required RMP amplitude for field penetration is usually much higher than that for mode locking, ${ }^{8,12}$ and the mode locking threshold is inversely proportional to the square of the island width. ${ }^{28}$ It is therefore expected that while the amplitude of the main component, having the same mode numbers as those of the NTM, is large enough to cause a non-uniform NTM rotation, the other sideband with lower amplitude might still be too weak to cause field penetration.

It should be mentioned that the diamagnetic drift effect has not been included in our model, which is known to significantly affect the island stability when the island width is sufficiently small. The ion polarization current associated the diamgnetic drift together with the transport threshold lead to a marginal island width above which the NTM can grow. ${ }^{31,32,41-44}$ Although the transport threshold due to the finite ratio between the parallel and perpendicular heat transport coefficients has been self-consistently included in our calculations, which also leads to a marginal island width, the marginal island width would be smaller when further taking into account the stabilizing effect of the diamgnetic drift and the associated ion polarization current. ${ }^{31,32,41-44}$ For the NTM stabilization by combined ECCD and RMP studied here, only the stabilization of a large island, being larger than the critical island width to flatten the plasma pressure profile across the island ${ }^{31}$, is of concern. In this case the diamgnetic drift effect and the associated ion polarization current are weak, and the island width is approximately determined by the bootstrap current density perturbation, the tearing mode stability index and the rf current. ${ }^{18,32 \text {, }}$ 41, 43 Therefore, the single fluid equations utilized in this paper are approximately valid for the island width being larger than the critical island width, which is about a few percent of the plasma minor radius in existing tokamaks and even smaller in a 
fusion reactor. ${ }^{31,32,41-44}$ The diamagnetic effect, being important for a smaller island, needs to be examined in the future by using two-fluid equations.

In summary, the stabilization of NTM by ECCD is found to be enhanced by a static RMP if the applied RMP phase has a half period difference from that of the ECW deposition region along helical angle. About one third rf current can be saved for mode stabilization, if an appropriate RMP amplitude is applied. The maximum enhancement is obtained for the RMP amplitude slightly below the mode locking threshold. If there is a feedback control of the RMP amplitude, then mode locking can be avoid.

\section{Acknowledgement}

This work is supported by the National Magnetic Confinement Fusion Science Program of China (Contract No. 2012GB103000 and 2012GB103002)and the National Natural Science Fundation of China (Contract No.11175211).

\section{Reference}

1. Z. Chang, J. D. Callen, E. D. Fredrickson, R. V. Budny, C. C. Hegna, K. M. McGuire and M. C. Zarnstorff, Physical review letters 74, 4663-4666 (1995).

2. A. Isayama, Y. Kamada, T. Ozeki and N. Isei, Plasma Physics and Controlled Fusion 41, 35-43 (1999).

3. O. Sauter, R. J. LaHaye, Z. Chang, D. A. Gates, Y. Kamada, H. Zohm, A. Bondeson, D. Boucher, J. D. Callen, M. S. Chu, T. A. Gianakon, O. Gruber, R. W. Harvey, C. C. Hegna, L. L. Lao, D. A. Monticello, F. Perkins, A. Pletzer, A. H. Reiman, M. Rosenbluth, E. J. Strait, T. S. Taylor, A. D. Turnbull, F. Waelbroeck, J. C. Wesley, H. R. Wilson and R. Yoshino, Physics of Plasmas 4, 1654-1664 (1997).

4. H. Zohm, D. A. Gates, H. R. Wilson, G. Gantenbein, O. Gruber, S. Gunter, M. Maraschek, A. W. Morris, M. Sokoll and D. Wagner, Plasma Physics and Controlled Fusion 39, B237 (1997).

5. S. Günter, A. Gude, M. Maraschek, Q. Yu and t. A. U. Team, Plasma Physics and Controlled Fusion 41, 767-774 (1999).

6. R. J. Buttery, S. Gunter, G. Giruzzi, T. C. Hender, D. Howell, G. Huysmans, R. J. La Haye, M. Maraschek, H. Reimerdes, O. Sauter, C. D. Warrick, H. R. Wilson and H. Zohm, Plasma Physics and Controlled Fusion 42, B61-B73 (2000). 7. R. J. La Haye, L. L. Lao, E. J. Strait and T. S. Taylor, Nuclear Fusion 37, 397 (1997).

8. R. FITZPATRICK, NUCLEAR FUSION 33, 1049-1084 (1993).

9. M. F. F. Nave and J. A. Wesson, Nuclear Fusion 30, 2575-2583 (1990).

10. B. Esposito, G. Granucci, M. Maraschek, S. Nowak, A. Gude, V. Igochine, E. Lazzaro, R. McDermott, E. Poli, J. Stober, W. Suttrop, W. Treutterer, H. Zohm and D. Brunetti, Nuclear Fusion 51, 083051 (2011).

11. Q. Yu, S. Günter and K. H. Finken, Physics of Plasmas 16, 042301 (2009). 
12. T. C. Hender, R. Fitzpatrick, A. W. Morris, P. G. Carolan, R. D. Durst, T. Edlington, J. Ferreira, S. J. Fielding, P. S. Haynes, J. Hugill, I. J. Jenkins, R. J. Lahaye, B. J. Parham, D. C. Robinson, T. N. Todd, M. Valovic and G. Vayakis, Nuclear Fusion 32, 2091-2117 (1992).

13. C. C. Hegna and J. D. Callen, Physics of Plasmas 4, 2940 (1997).

14. H. Zohm, Physics of Plasmas 4, 3433-3435 (1997).

15. Q. Yu, S. Günter, G. Giruzzi, K. Lackner and M. Zabiego, Physics of Plasmas 7, 312 (2000).

16. Q. Yu, X. D. Zhang and S. Günter, Physics of Plasmas 11, 1960 (2004).

17. O. Sauter, Physics of Plasmas 11, 4808-4813 (2004).

18. R. J. La Haye, Physics of Plasmas 13, 055501-055501-055518 (2006).

19. G. Gantenbein, H. Zohm, G. Giruzzi, S. Günter, F. Leuterer, M. Maraschek, J. Meskat, Q. Yu, A. U. Team and E.-G. (AUG), Physical Review Letters 85, 12421245 (2000).

20. C. C. Petty, R. J. La Haye, T. C. Luce, D. A. Humphreys, A. W. Hyatt, J. Lohr, R. Prater, E. J. Strait and M. R. Wade, Nuclear Fusion 44, 243-251 (2004).

21. A. Isayama, G. Matsunaga, T. Kobayashi, S. Moriyama, N. Oyama, Y. Sakamoto, T. Suzuki, H. Urano, N. Hayashi, Y. Kamada, T. Ozeki, Y. Hirano, L. Urso, H. Zohm, M. Maraschek, J. Hobirk, K. Nagasaki and t. J.-. team, Nuclear Fusion 49, 055006 (2009).

22. G. P. Canal, B. P. Duval, F. Felici, T. P. Goodman, J. P. Graves, A. Pochelon, H. Reimerdes, O. Sauter, D. Testa and t. T. Team, Nuclear Fusion 53, 113026 (2013).

23. H. Zohm, G. Gantenbein, F. Leuterer, A. Manini, M. Maraschek and Q. Yu, Nuclear Fusion 47, 228-232 (2007).

24. R. J. La Haye, R. Prater, R. J. Buttery, N. Hayashi, A. Isayama, M. E. Maraschek, L. Urso and H. Zohm, Nuclear Fusion 46, 451 (2006).

25. D. De Lazzari and E. Westerhof, Nuclear Fusion 49, 075002 (2009).

26. W. Wehner and E. Schuster, Nuclear Fusion 52, 074003 (2012).

27. M. Maraschek, G. Gantenbein, Q. Yu, H. Zohm, S. Günter, F. Leuterer, A. Manini, E. Group and A. U. Team, Physical Review Letters 98, 025005 (2007).

28. Q. Yu and S. Günter, Nuclear Fusion 48, 065004 (2008).

29. Q. Hu, B. Rao, Q. Yu, Y. Ding, G. Zhuang, W. Jin and X. Hu, Physics of Plasmas 20, 092502 (2013).

30. Q. Yu, X. D. Zhang and S. Günter, Physics of Plasmas 11, 1960 (2004).

31. R. Fitzpatrick, Physics of Plasmas 2, 825 (1995).

32. O. Sauter, R. J. Buttery, R. Felton, T. C. Hender, D. F. Howell and c. t. t. E.J. Workprogramme, Plasma Physics and Controlled Fusion 44, 1999-2019 (2002).

33. F. L. Waelbroeck, Nuclear Fusion 49, 104025 (2009).

34. F. A. G. Volpe, M. E. Austin, R. J. La Haye, J. Lohr, R. Prater, E. J. Strait and A. S. Welander, Physics of Plasmas 16, 102502-102502-102514 (2009).

35. J. Jacquinot, S. Putvinski, G. Bosia, A. Fukuyama, R. Hemsworth, S. Konovalov, W. M. Nevins, F. Perkins, K. A. Rasumova, F. Romanelli, K. Tobita, K. Ushigusa, J. W. Van Dam, V. Vdovin, S. Zweben, M. Makowski, R. Koch, J. G. 
Wegrowe, V. V. Alikaev, B. Beaumont, A. Becoulet, S. Bernabei, V. P. Bhatnagar, S. Bremond, M. D. Carter, C. D. Challis, A. Cote, D. A. D'Ippolito, F. Engelmann, L. G. Eriksson, N. Fujisawa, G. Giruzzi, C. Gormezano, R. Harvey, J. Heikkinen, T. Hellsten, Y. Ikeda, T. T. C. Jones, H. Kimura, B. Lloyd, T. Luce, D. Moreau, O. Naito, F. Nguyen, J. M. Noterdaeme, W. Ott, C. C. Petty, A. Polevoi, R. Prater, J. Rogers, F. Santini, F. X. Soldner, D. Stork, E. Thompson, G. Tonon, A. Tuccillo, E. Westerhof, H. Zohm, D. E. Post, N. A. Uckan, M. Azumi, D. J. Campbell, N. Ivanov, N. Sauthoff, M. Wakatani, W. M. Nevins and E. Righi, Nuclear Fusion 39, 2495-2539 (1999).

36. T. E. Evans, M. E. Fenstermacher, R. A. Moyer, T. H. Osborne, J. G. Watkins, P. Gohil, I. Joseph, M. J. Schaffer, L. R. Baylor, M. Bécoulet, J. A. Boedo, K. H. Burrell, J. S. deGrassie, K. H. Finken, T. Jernigan, M. W. Jakubowski, C. J. Lasnier, M. Lehnen, A. W. Leonard, J. Lonnroth, E. Nardon, V. Parail, O. Schmitz, B. Unterberg and W. P. West, Nuclear Fusion 48 (2) (2008).

37. Y. Liang, C. G. Gimblett, P. K. Browning, P. Devoy, H. R. Koslowski, S. Jachmich, Y. Sun and C. Wiegmann, Physical Review Letters 105 (6) (2010).

38. W. Suttrop, T. Eich, J. C. Fuchs, S. Günter, A. Janzer, A. Herrmann, A. Kallenbach, P. T. Lang, T. Lunt, M. Maraschek, R. M. McDermott, A. Mlynek, T. Pütterich, M. Rott, T. Vierle, E. Wolfrum, Q. Yu, I. Zammuto and H. Zohm, Physical Review Letters 106 (22) (2011).

39. Q. Yu and S. Günter, Nuclear Fusion 49, 062001 (2009).

40. F. L. Waelbroeck, I. Joseph, E. Nardon, M. Bécoulet and R. Fitzpatrick, Nuclear Fusion 52, 074004 (2012).

41. H. R. Wilson, J. W. Connor, R. J. Hastie and C. C. Hegna, Physics of Plasmas 3, 248-265 (1996).

42. R. J. La Haye and O. Sauter, Nuclear Fusion 38, 987-999 (1998).

43. F. L. Waelbroeck, J. W. Connor and H. R. Wilson, Physical Review Letters 87 (2001).

44. Q. Yu, Nuclear Fusion 50, 025014 (2010). 